\title{
Pharmacokinetic and Pharmacodynamic Modelling in Paediatric Bone Marrow Transplantation
}

\author{
Elham Alshammari* \\ Department of Pharmacy Practice, Faculty of Pharmacy, Princess Nourah bint Abdul Rahman University, Riyadh, \\ Saudi Arabia
}

Received: $17^{\text {th }}$ Dec, 18; Revised: $16^{\text {th }}$ Jan, 19, Accepted: $18^{\text {th }}$ Feb, 19; Available Online: $25^{\text {th }}$ Mar, 2019

\begin{abstract}
Building pharmacokinetics and pharmacodynamics models that describe the relationship between drug concentration and its clinical response in different disease conditions and age groups should be emphasized. This review aims to explore modelling in case of bone marrow transplantation staring from bone marrow and immune system, transplantation process, immune reconstitution and graft versus host disease, the difference in case of children, to handling of data that arise from patients.
\end{abstract}

Keywords: pharmacokinetics, pharmacodynamics, bone marrow, transplantation, children. Bone marrow transplantation.

\section{INTRODUCTION}

The bone marrow contains pluripotent stem cells and postthymic lymphocytes, which are responsible for body immunity through unique defensive mechanisms. Bone marrow transplant (BMT) or Hematopoietic cell transplant (HCT) is the infusion of hematopoietic cells into a patient to treat or restore normal haematopoiesis and lymphopoiesis following depletion of host cells using chemotherapy and/or radiotherapy. ${ }^{1}$ Examples of conditions where BMT is used: Cancers such as leukaemia, lymphoma, and multiple myeloma. A disease that affects the production of bone marrow cells, such as: sickle cell anaemia, aplastic anaemia, thalassemia, congenital neutropenia, and severe immunodeficiency syndromes. Previous chemotherapy that destroyed bone marrow. There are three kinds of BMT: Autologous BMT where Stem cells are removed from same subject before receiving chemotherapy and/or radiation treatment. Allogeneic BMT where stem cells are removed from a donor. In acute myeloid leukaemia (AML) the percentage survival was comparable to autologous BMT while percentage relapse was $50 \%$ less. $^{2}$ Umbilical cord blood transplant where stem cells are removed from a newborn baby's umbilical cord immediately after being born and then stored until they are needed for a transplant. Immune system and maturation Background Bone marrow is the source of haematopoietic stem cells, the precursor of all blood cells (white blood cells (WBC), red blood cells, and platelets) and has the capacity to replicate indefinitely. White blood cells, Leucocyte, responsible for immunity can be classed depending on the presence of granules into granulocyte (neutrophils, basophils, and eosinophils) and agranulocyte (lymphocytes, monocytes, and macrophages). ${ }^{3}$ Lymphocytes Lymphocytes, represent $40 \%$ of WBC, are the major cells involved in antigen- specific immune responses. They can be distinguished by clusters of differentiation (CDs) which are antigen-specific surface receptors. The two major types of lymphocyte are $\mathrm{T}$ and $\mathrm{B}$ cells.

T-lymphocytes (T-cells)

$\mathrm{T}$ - cells which mature in the thymus are the most predominant type. There are major subclasses of $\mathrm{T}$-cells identified, cytotoxic T-cells (CD8+), helper T-cells (CD4+) cells, regulatory T-cells and natural killer (NK) Tcells. Helper T-cells in response to stimuli secret cytokines that activate other T-cells and leucocytes (including Bcells) to differentiate and expand. Regulatory T-cells main function is to inhibit this response. NK cells, involved in the innate immunity, unlike other T-cells they kill infected cells without the need for expression of antigen protein in their surface.

\section{Cytokines}

Cytokines, molecules secreted from CD4+ and CD8+ upon activation, are linked with GvHD in the following ways. There are two isolates of cytokines; The first named cytokine type I (proinflammatory) that activate GvHD while cytokine type II (anti-inflammatory) inhibits GvHD. ${ }^{4}$

B-lymphocytes (B-cells)

B-cells which mature in bone marrow can present antigen to T-cells. Their main function is to secrete antibodies when developed into plasma cells.

Neutrophils

Neutrophils represent $60 \%$ of granulocytes, considered the first defence line against different pathogens and inflammatory processes, and have a key role in immune response through phagocytosis (ingestion of invaders) and forming immunomodulator agents that are sometimes called chemoattractant (cytokines and chemokines) eventually recruiting more immune cells. The decrease in 
circulating neutrophils after treatment with chemotherapy is expected and neutropenia can be defined by absolute neutrophil count (ANC) below $1.0 \times 109 / \mathrm{L}^{5}$

There are two types of immunity Innate immunity developed in early life and the basic components of this type are; phagocytes (neutrophils and macrophages), and natural killer cells (NK). Adaptive immunity developed in the later response to antigens and the basic components of this type are; T-cells (cell mediated immunity), and B-cells and their secreted antibodies (humoral immunity).

Interaction of both types to get rid of invaders, is called the effector phase, after which a second phase begins, homeostasis, when lymphocytes die by a process known as apoptosis in order to retain normal immune response. Another important type of cells is antigen presenting cells (APC) or dendritic cells (different APCs specialized for Bcells are also present). Their main function is to capture and transport antigens to naïve T-cells in lymphoid organs where it will be again differentiated and expanded. A small portion of these differentiated naïve cells will remain as memory T-cells although some of these differentiated naïve cells may preserve their naïve phenotype for weeks. The majority of memory T-cells, compared to naïve Tcells, are considered short-lived. The turnover rate for naive $\mathrm{T}$-cell takes years while it may take months for memory T-cell. ${ }^{6-8}$

Immune reconstitution and graft versus host disease (GvHD)

The success of engraftment is related to the type of conditioning regimen used prior to BMT and restoring normal immune reconstitution. The conditioning regimen is designed to suppress the immunity to control GvHD (e.g. immunosuppressants), eradicate residual malignancy, or to create space in the marrow compartment. Abnormal immune reconstitution and related graft versus host disease (GvHD) is the major concern after BMT. GvHD is divided into two types; acute and chronic GvHD. Acute GvHD usually occurs in the first three months after BMT affecting skin, liver, and gastrointestinal tract (GIT). Chronic GvHD occurs after three months affecting any organ system similar to autoimmune diseases and could be further divided into delayed (up to 2 years), late (up to 10 years), and very late (>10 years). GvHD provides an environment that favours opportunistic infections with cytomegalovirus pneumonitis (CMV-P) being the most frequent and severe. The combination of GvHD and infections are leading causes of mortality in BMT patients. $^{9-12}$

A survey included experience with therapies for chronic GvHD from 188 adult and paediatric transplantation programs concluded that $28 \%$ had success when used systemic corticosteroids, $13 \%$ had similar success with cyclosporin or mycophenolate mofetil. ${ }^{13}$

Immune reconstitution in children

In general children have a more naïve immune system and tend to recover better and faster than adults after BMT. Mobilization of lymphocytes from marrow to thymus decreases with aging. Because those sick children have abnormal immunity before infusing stem cells and receive different regimen of immunosuppressant agents after BMT, the timing for healthy reconstitution will vary. Three months are usually required for innate immunity to recover while adaptive immunity, especially CD4+ cells, takes longer. Delay in immune reconstitution of up to two years, is expected, because of chronic GvHD and its treatment. ${ }^{14}$ It is expected for recovery from neutropenia to take place within three weeks, if more this is first indicator of unsuccessful BMT. T-cells as a whole mainly recover within 6 months but the type of all depends on the subset: CD8+ takes months while for CD4+ takes longer, up to years. Recovery of B-cells takes longer than T-cells (up to 2 years). Recovery of APCs take 1 month up to 1 year ${ }^{4,15}$. Transplant process

The process of transplantation can be summarized as follows:

Admission at least 1 week before BMT (day -7) where the patient receives either chemotherapy or/and radiotherapy to deplete unhealthy cells and help engraftment.

At day 0 of BMT (day 0);

Intravenous infusion of stem cells; CD3+ T-cells that are responsible for $\mathrm{T}$-cell activation and $\mathrm{CD} 34+$ the selfrenewing hematopoietic stem cells and sometimes called progenitors. The dose is between $1-10 \times 10^{6} \mathrm{CD} 34+/ \mathrm{kg}[2$, 4],

Giving immunosuppressant agents and other agents (antibacterial, antifungal, antiviral) to help guard against rejecting the new graft and GvHD.

New stem cells mobilize to the marrow where it can mature and differentiate to form healthy cells. Sometimes administration of cytokine granulocyte colony-stimulating factor (G-CSF), a mobilizing agent that speeds up the recovery by few days, is used.

Pharmacometrics

Pharmacokinetics has been defined as the time course of drug concentration in the body while pharmacodynamics (PD) is the study of the time course of pharmacological effects of these drugs. ${ }^{16,17}$ In 1982 the editors of journal of pharmacokinetics and biopharmaceutics used pharmacometrics as a scientific term. Pharmacometrics is "the science of developing and applying mathematical and statistical methods to characterize, understand, and predict a drug's pharmacokinetic, pharmacodynamic, and biomarker-outcomes behaviour". ${ }^{18}$ Source of variabilityFor pharmacokinetic results to be generalized, factors affecting variability must be considered. Examples of these factors include: Demographics such as gender, body weight, body surface area (BSA), age, and raceGenetic phenotype affecting the hepatic metabolism of drugs via cytochrome P450 isoforms (e.g., CYP2D6, 2C19, 2C9, 2A6), Drug-drug interactions, and Disease states and pathophysiological factors affecting renal and hepatic impairment.Nature of variables effectVariability is usually characterized in terms of fixed and random effects. Fixed effect

Those variables are fixed in nature (e.g. dose, time of sampling, male/female, population PK average parameters)

Random effect 
The random effects quantify the amount of pharmacokinetic variability that is not explained by the fixed effects and do not affect the result of the experiment. These random effects subsets are

Inter-individual variability.

Interindividual variability in pharmacokinetics is viewed incorrectly by many as a nuisance factor that must be overcome through complex study designs and inclusion criteria. However, as PK data are usually collected in patients, it is important to account for inter-individual difference in model parameters.

Residual variability.

Residual variation includes:

Intra-individual or inter-occasion variability (random changes in a patient's parameter values over time or from one occasion to another),

Drug concentration measurement assay error.

Model misspecification, that is the difference between model prediction and observation after accounting for other sources of variability.

Mixed effect

Any model that contains both fixed and random effects is mixed effect model. ${ }^{18-21}$

PKPD modelling guidance published by FDA emphasises the importance of describing the relationship between drug concentration and clinical response of licensed drugs but in different population group (e.g. children). ${ }^{22}$

Approaches to handling PKPD data

There are three common approaches to modelling data that arise from patients, who can be considered experimental units that differ from each other.

Nä̈ve pooled

In this approach, time concentration data are pooled together as if all doses and all observations belong to a single subject. No information is available on individual subject parameters. This approach may be satisfactory if data are extensive for each subject and if there is only minor between-subject variability. A major disadvantage is that inter-individual and residual variability cannot be separated. ${ }^{23}$

Two-stage

In the two-stage approach each subject's PK parameters have to be calculated using regression methods. These are then summarized. The main disadvantage with the twostage method is that extensive data is required from every subject so that their parameters can be calculated. ${ }^{18,23}$ Mixed effect

There are two types of mixed effect models; linear and nonlinear.

\section{Linear mixed effect models (LMEM)}

The problem in two-stage approach could be avoided through Linear mixed effect models (LMEM). LMEM are not commonly seen in analysis of PK data but more commonly seen in analysis of PD data. It is assumed that any covariate (e.g. age, wt, CLcr) is constant but in mixed effect models it becomes time-varying. ${ }^{18}$

Nonlinear mixed effect modelling (NLMEM)

The difference between nonlinear mixed effect models from linear mixed effect models is that the function under consideration is nonlinear in the model parameter, as in the area with many PK models. NLMEM can pool rich and sparse data to provide estimation of typical population parameters along with their variance. This method allows all of the data to contribute, without losing the concept that it comes from different individuals. NLMEM methods are established as the optimal method for analysing PK data. ${ }^{18}$ Type of Models

Overview

Whilst it is now clear that NLMEM is the best technique to fit PKPD data, it is also important to consider the types of models to be fitted. Pharmacokinetics can be largely descriptive without adequate understanding of the covariates, or physiology which gives rise to the data. ${ }^{24}$ PK Models representing system under study can be classified into two types: models of data (empirical models) and models of systems (mechanistic models). ${ }^{18}$

Empirical models

Although empirical models are not completely nonmechanistic, few assumptions are made where there is little information about data and nature of process generating them. Allometric scaling ${ }^{23}$ and sum of exponentials describing concentration-time profile are examples of assumptions.

Mechanistic models

Mechanistic models are based on physical and physiological principles considering factors such as receptor binding, organ blood flow and volumes. Mechanistic models can be categorized into types such as time variant (one parameter changing over time), stochastic (adding prior to the model), linear or nonlinear. Model building and selection

In general, physiological systems are complex and nonlinear. The model should be simple (reduce the model as possible), producing clinically significant findings, reliable, and parameter estimates are reproducible with different dataset. The basic strategy is to decide which random effects to be included, structure variancecovariance models compare between them using statistical and graphical software, and then select best model among them.

Using NONMEM software, the selection of any parameter depends on comparing the minimum value of objective function (OFV) generated from the software output (decrease by 3.85 define the significance by $\mathrm{p}$-value $<0.05)$.

Goodness of fit (GOF) graphical check which is scattered plots with trend line i.e. observation vs. Prediction (predicted value from the model), observation vs. Individual prediction, conditional weighted residuals (CWRES) vs. Time, and CWRES vs. Prediction. ${ }^{18}$

Bootstrap methods (simulation of data) used to evaluate parameter uncertainty. In order to estimate $95 \%$ confidence intervals, one thousand iterations may be required. Then compare simulated data from the actual observations.

Another method is to use visual predictive check (VPC) graphs. $90 \%$ prediction intervals generated from the VPC should capture $90 \%$ of the observations. ${ }^{23}$

PKPD modelling of Monoclonal antibody (Moab) in 
children undergoing BMT

Moab target mediated disposition

PKPD modelling of target mediated drugs is complex. Non-compartmental analysis (NCOMP) as a useful tool for obtaining drug profile overview but not for understanding dose-response relationship. This is because NCOMP only describes the observed data, it does not generate parameters with biological meaning using dose and concentration. This is especially important with drugs like Moab target mediated disposition where the elimination occurs not from systemic circulation but from tissues via receptor mediated endocytosis.

Modelling the time course of the drug PK and relationship to the drug effect PD will provide predictive tool for better treatment. ${ }^{25}$

\section{CONFLICT OF INTEREST AND FUNDING}

The author declares any conflict of interest or source of funding.

\section{REFERENCES}

1. Greene, R.J. and N.D. Harris, Major pathological process in disease. In: Pathology and Therapeutics for Pharmacists Abasis for clinical pharmacy practice. 3rd ed. 2008, London and Chicago: Pharmaceutical Press.

2. Sell, S., Stem cells handbook. 2004: Humana Press. 509.

3. Janeway, C.A., P. Travers, and M. Walport, Immunobiology: The Immune System in Health and Disease. 5th edition ed. 2001, New York: Garland Science.

4. Cottler-Fox, M.H., et al., Stem Cell Mobilization. hemtology, 2003. (1): p. 419-437.

5. Cassatella, M.A., The neutrophil: an emerging regulator of inflammatory and immune response. 2003: Karger Publishers.

6. Abbas, K.A., A.H. Lichtman, and S. Pillai, Cellular and Molecular Immunology. 2010: Elsevier Health Sciences.

7. Tough, D.F. and J. Sprent, Turnover of Naive- and Memory-phenotype T Cells. the journal of experimental medicine, 1994 179: p. 1127-1135.

8. Asquith, b., et al., Lymphocyte kinetics in health and disease. Trends in Immunology, 2009. 30: p. 182-189.

9. Jacobsohn, D.A., Acute graft-versus-host disease in children. Bone Marrow Transplantation, 2008. 41: p. 215-221.

10. Woo, S.B., S.J. Lee, and M.M. Schubert, GRAFT-VS.HOST DISEASE. Critical Reviews in Oral Biology \& Medicine, 1997. 8(2): p. 201-216.

11. Mohty, M. and J.F. Apperley, Long-Term Physiological Side Effects After Allogeneic Bone
Marrow Transplantation. hematology, 2010: p. 22936.

12. Barry, S.M., M.A. Johnson, and G. Janossy, Cytopathology or immunopathology? The puzzle of cytomegalovirus pneumonitis revisited. Bone Marrow Transplant, 2000. 26(6): p. 591-7.

13.Lee, S.J., et al., A survey of diagnosis, management, and grading of chronic GVHD. Biology of blood and marrow transplantation, 2002. 8(1): p. 32-9.

14. Koehl, U., et al., Immune recovery in children undergoing allogeneic stem cell transplantation: absolute CD8bCD3p count reconstitution is associated with survival. Bone Marrow Transplantation, 2007. 39: p. 269-278.

15. Trigg, M.E., Hematopoietic Stem Cells. Pediatrics, 2004. 113(4): p. 1051-7.

16. Wagner, J.G., HISTORY OF PHARMACOKINETICS. Pharmacology and Therapeutics, 1981. 12(3): p. 537562.

17. Mager, D.E., E. Wyska, and W.J. Jusko, Diversity of Mechanism-Based Pharmacodynamic Models. Drug metabolism and disposition, 2003. 3(31): p. 510-519.

18. Bonate, P.L., Pharmacokinetic-Pharmacodynamic Modeling and Simulation. 2nd ed. 2011, IL: Springer. 1-637.

19.Ette, E. and P. Williams, Population Pharmacokinetics I: Background, Concepts, and Models. Annal of Pharmacotherapy, 2004. 38: p. 1702-6.

20. Ette, E. and P. Williams, Population Pharmacokinetics II: Estimation Methods. Annal of Pharmacotherapy, 2004. 38: p. 1907-15.

21.Ette, E., P. Williams, and J.R. Lane, Population Pharmacokinetics III: Design, Analysis, and Application of Population Pharmacokinetic Studies. Annal of Pharmacotherapy, 2004: p. 2136-44.

22. US Food and Drug Administration, Guidance for Industry Exposure-Response Relationships - Study Design, Data Analysis, and Regulatory Applications. 2003: Rockville, MA, USA.

23. Anderson, B.J., K. Allegaert, and N.H.G. Holford, Population clinical pharmacology of children: general principles. European Journal of Pediatrics, 2006. 11(165): p. 741-6.

24. Steimer, J., et al., Modelling the genesis and treatment of cancer: The potential role of physiologically based pharmacodynamics. European Journal of Cancer, 2010. 46: p. 21-32.

25.Zhiqiang, A., Therapeutic Monoclonal Antibodies: From Bench to Clinic. 2011, John Wiley and Sons,. p. 896. 